\title{
Resource Utilization and Costs Associated with Using Insulin Therapy Within a Newly Diagnosed Type 2 Diabetes Mellitus Population
}

\author{
Kelly Bell, PharmD, MSPhr; Shreekant Parasuraman, PhD; Aditya Raju, BPharm, MS; \\ Manan Shah, PharmD, PhD; John Graham, PharmD; and Melissa Denno, PharmD, MS
}

\begin{abstract}
BACKGROUND: Although oral antidiabetic medications are the mainstay for managing type 2 diabetes mellitus (T2DM), patients often require insulin therapy to achieve optimal glycemic control. Given the prevalence of insulin use among patients with T2DM, this study evaluated the economic impact of this treatment modality in patients treated in a managed care setting.
\end{abstract}

OBJECTIVE: To estimate costs and resource utilization associated with using insulin therapy among patients with newly diagnosed T2DM who were initially treated with other noninsulin antidiabetic (NIAD) medications.

METHODS: An observational, retrospective study design was implemented using integrated medical and pharmacy claims data. Adults with a diagnosis of T2DM from July 1, 2003, through March 31, 2008, were identified. The date of first diagnosis was deemed the index date. The 24-month period after the index date was used to assess treatment patterns. Based on the treatment patterns, the following 2 cohorts were selected: NIAD-only cohort, users who received $>1$ NIAD class medication but never received insulin, and insulin-use cohort, NIAD users who switched to/added on insulin therapy (duration $\geq 60$ days). Patients were matched in a 1:3 (insulin-use:NIADonly) ratio based on propensity scores and other key covariates of interest. Hypoglycemia rates, monthly costs, and resource use during the outcome assessment period were compared between cohorts.

RESULTS: After matching, 1,400 patients (350 insulin users and 1,050 NIADonly users) were included in the analysis ( $42 \%$ women; mean age, 56 years). After controlling for covariates, the insulin-use cohort incurred $\$ 71$ per patient per month higher total T2DM-specific costs than the NIAD-only cohort (\$241/month vs. \$170/month, $P=0.0003$ ). Pharmacy costs and utilization of physician visits were drivers of cost differences between cohorts. The rate of hypoglycemic events was 10.2 per 100 person-years for the insulin-use cohort versus 2.9 per 100 person-years in the NIAD-only cohort $(P<0.0001)$.

CONCLUSIONS: Use of insulin therapy is associated with increased hypoglycemic events, increased pharmacy and medical costs, and greater utilization of T2DM-specific health care services.

J Manag Care Spec Pharm. 2015;21(3):220-28

Copyright @ 2015, Academy of Managed Care Pharmacy. All rights reserved.

\section{What is already known about this subject}

Insulin is often prescribed to patients with type 2 diabetes (T2DM) when glycemic control is inadequate with noninsulin antidiabetic (NIAD) therapy.

Several studies have demonstrated the comparable efficacy of insulin and oral antidiabetic agents in achieving target glycemic levels. However, there is limited evidence quantifying the economic impact of insulin use in patients with T2DM.

\section{What this study adds}

The introduction of insulin therapy early in the treatment pathway was associated with increased T2DM-specific costs of $\$ 71$ per month per patient. These increased costs were driven mainly by higher pharmacy costs and a higher number of physician visits. Inclusion of insulin therapy in the treatment regimens of patients with T2DM was associated with an approximately 4-fold increase in rates of hypoglycemic events compared with treatment regimens that include NIAD therapies only.

T ype 2 diabetes mellitus (T2DM) is a chronic disease commonly associated with serious microvascular and macrovascular complications. It is associated with high morbidity and mortality, resulting in increased health care resource utilization and costs. The American Diabetes Association (ADA) estimated that the total costs (direct and indirect) associated with managing diabetes were $\$ 245$ billion in 2012. ${ }^{1}$ Furthermore, in 2012, although people with diabetes accounted for only $7 \%$ of the U.S. population, more than 1 in 5 health care dollars were spent caring for diabetic patients. ${ }^{1}$ Using age- and sex-adjusted data, the ADA also reported that people with diabetes had annual health care expenditures of approximately $\$ 13,741$, whereas the same population without diabetes had annual health care expenditures of approximately $\$ 5,853$. Therefore, annual excess health care expenditures of approximately $\$ 7,888$ per patient could be attributed to diabetes. ${ }^{1}$

Diabetes care is complex and requires that many issues be addressed. Achieving long-term glycemic control is key in the management of diabetes and in the reduction and prevention of complications. ${ }^{2}$ Treatment guidelines include lifestyle modifications, noninsulin oral antidiabetic (NIAD) therapy, and/or insulin therapy for achieving target fasting plasma glucose and glycated hemoglobin (Alc) levels. However, use of metformin, together with lifestyle modifications, is recommended as firstline treatment before the addition of another NIAD or insulin for most patients. ${ }^{3,4}$ Insulin use has also been shown to have rates of glycemic control similar to oral alternatives.$^{5-7}$ Lingvay et al. (2009) found that patients taking an oral regimen (metformin, pioglitazone, and glyburide) had Alc levels similar to those of patients who were taking insulin and metformin. ${ }^{5}$ Likewise, findings from 2 meta-analyses suggest that add-on 
insulin therapy or oral agents produce comparable results with respect to Alc reduction. ${ }^{6,7}$ Furthermore, patients who use insulin or medication combinations that include insulin are at higher risk for hypoglycemic events (plasma glucose $<70$ milligrams per deciliter) than patients who do not use insulin..$^{8-11}$ Henderson et al. (2003) reported that $73 \%$ of patients had experienced hypoglycemia since starting insulin treatment and that the risk of hypoglycemia increased with increasing duration of diabetes and insulin therapy. ${ }^{8}$ Hypoglycemia is associated with adverse short- and long-term outcomes, such as increased mortality, seizures, and coma. ${ }^{12-16}$ In addition, fear of hypoglycemia can lead to medication noncompliance and failure to achieve glycemic control. ${ }^{12,15}$

Given the similarities between the effects of oral and insulin-containing antidiabetic therapies on glycemic control and the risk of hypoglycemia with insulin, there is a need to assess the impact of adding insulin to a T2DM regimen on outcomes of health care costs and resource use. Limited evidence exists for these outcomes ${ }^{17}$ so the purpose of this study was to assess health care resource utilization and costs associated with the use of insulin therapy for the treatment of T2DM. Specifically, the aim was to evaluate the costs, resource utilization, and hypoglycemia rates associated with the use of insulin therapy among newly diagnosed patients with T2DM receiving NIAD therapy in a managed care setting.

\section{Methods}

\section{Data Source}

An integrated source of medical and pharmacy claims from the IMS LifeLink Health Plans Claims Database was used for this study. This database included longitudinal, integrated, and patient-level medical and pharmaceutical claims comprising 5 billion patient observations from across the United States for more than 70 million patients from more than 100 health plans, including medical services and prescription drug information across the entire continuum of care. Specifically, inpatient and outpatient diagnoses (by International Classification of Diseases, Ninth Revision, Clinical Modification [ICD-9-CM] diagnosis codes) and procedures (in Current Procedural Terminology, Version 4, and Healthcare Common Procedural Coding System formats) and retail and mail-order prescription records, which include National Drug Code numbers and the quantity dispensed, were available for all patients. Cost/charge information and dates of service were available for all services rendered. The data were fully de-identified and compliant with the Health Insurance Portability and Accountability Act of 1996.

\section{Study Design and Patient Population}

The analysis time frame for this observational, retrospective cohort study ranged from January 1, 2003, through March 31, 2010. Patients aged at least 18 years with a diagnosis of T2DM

\section{TABLE 1 Antidiabetic Therapies}

\begin{tabular}{l|l}
\hline Antidiabetic Therapy & \multicolumn{1}{c}{ Drug Name } \\
\hline Insulin & $\begin{array}{l}\text { Insulin aspart, insulin aspart protamine and } \\
\text { aspart (human), insulin detemir, insulin } \\
\text { glargine, insulin glulisine, insulin isophane, } \\
\text { insulin isophane and regular (human), } \\
\text { insulin isophane (human), insulin isophane } \\
\text { (pork), insulin lispro (human), insulin lispro } \\
\text { protamine and lispro (human), insulin } \\
\text { regular (human) buffered, insulin regular }\end{array}$ \\
\hline Noninsulin medications & \multicolumn{2}{|l}{} \\
\hline Sulfonylureas & $\begin{array}{l}\text { Chlorpropamide, glipizide, glimepiride, } \\
\text { glyburide }\end{array}$ \\
\hline Meglitinides & Repaglinide, nateglinide \\
\hline Biguanides & Metformin \\
\hline DPP-4 inhibitors & Sitagliptin, saxagliptin \\
\hline Thiazolidinediones & Rosiglitazone, pioglitazone \\
\hline Alpha-glucosidase inhibitors & Acarbose, miglitol \\
\hline GLP-1 receptor agonists & Exenatide, liraglutide \\
\hline Amylin analogues & Pramlintide \\
\hline Combination drug products & $\begin{array}{l}\text { Metaglip (glipizide/metformin); Glucovance } \\
\text { (glyburide/metformin); Avandamet } \\
\text { (rosiglitazone/metformin); Actoplus Met } \\
\text { (pioglitazone/metformin); Avandaryl } \\
\text { (rosiglitazone/glimepiride); Duetact } \\
\text { (pioglitazone/glimepiride); Janumet } \\
\text { (sitagliptin/metformin) }\end{array}$ \\
\hline DDP-4=dipeptidyl peptidase-4; \begin{tabular}{l} 
GLP-1=glucagon-like peptide-1. \\
\hline
\end{tabular}
\end{tabular}

(ICD-9-CM codes $250 . x 0$ or 250.x2) and any evidence of diabetes drug therapy (including all types of insulin and NIAD drugs; Table 1) were the target population. The date of first T2DM diagnosis during the period from July 1, 2003, through March 31, 2008 (deemed the enrollment period) was defined as the index date. The 6-month period before the index date was defined as the pre-index period and was used to ensure that patients were (a) newly diagnosed and (b) new to antidiabetic medication. Receipt of antidiabetic therapy was assessed during the 24-month period after the index date and was defined as the post-index period. The date of the first antidiabetic medication during the post-index period was defined as the index prescription date (Figure 1).

Patients were required to be continuously eligible to receive medical and pharmacy services during the pre-index through the post-index periods. Patients were excluded if they had a diagnosis for type 1 diabetes mellitus (ICD-9-CM codes 250. $\mathrm{x} 1$ or 250.x3) or gestational diabetes (ICD-9-CM code 648.8x) during the analysis time frame or evidence of pregnancy (ICD9-CM codes 650.xx to 659xx or V22.2) during the pre-index period.

\section{Treatment Patterns and Group Definitions}

Antidiabetic therapy patterns of use were analyzed after the index prescription date, and patients were categorized into 7 types based on the following observed patterns: 


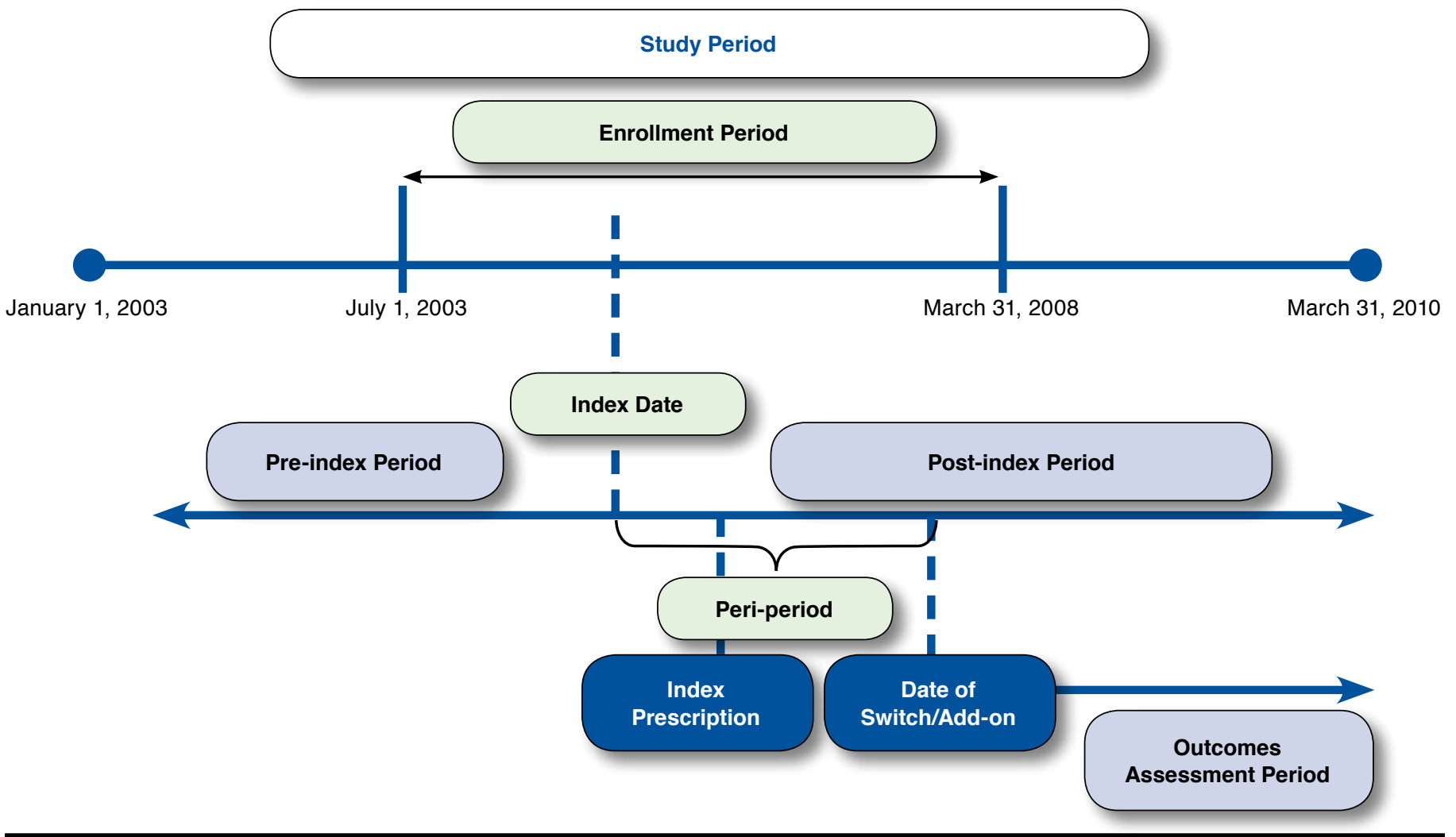

1. Noninsulin user: Patients who received NIAD therapy without any evidence of insulin therapy.

2. Insulin-only user: Patients using insulin without any evidence of NIAD therapy.

3. Early insulin user: Patients using insulin before starting NIAD therapy.

4. Same-day insulin user: Patients starting insulin and NIAD therapy on the same date.

5. Short-term insulin user: Patients who started insulin after NIAD therapy and whose total time on insulin was $<60$ days.

6. Intermittent insulin user: Patients who started insulin after NIAD therapy whose total time on insulin was $\geq 60$ days, but their medication possession ratio (MPR) was $<25 \%$.

7. Long-term insulin user: Patients who started insulin after NIAD therapy whose total time on insulin was $\geq 60$ days, but their MPR was $\geq 25 \%$.

As noted in this list, patients starting insulin after NIAD therapy were further classified as short-term, intermittent, and long-term. This classification was based on a combination of insulin duration and MPR because it is common for patients to discontinue insulin therapy due to various clinical issues.
Insulin duration was defined as the time between the first and last insulin prescription, and MPR was calculated as the ratio of days' supply of insulin to the total number of days between the first and last insulin prescriptions.

\section{Study Cohorts and Assessment of Outcomes}

To understand the specific impact of using insulin in patients with T2DM who initiated treatment with NIAD, the study cohorts were obtained from the noninsulin users and longterm insulin users described in the previous list, and this constituted the final population. Specifically, noninsulin users who first switched to/added on a second class of NIAD therapy were placed in the NIAD-only cohort, and long-term insulin users who first switched to/added on insulin after the initial NIAD therapy were placed in the insulin-use cohort. For this analysis, the 24-month post-index period was split into 2 periods: (1) the peri-period, defined as the time between the index date and date of first therapeutic class switch/ add-on, was used to capture disease severity proxy measures (T2DM-specific hospital/emergency room [ER] and T2DM-specific costs) and (2) the outcomes assessment period, defined as the time between the date of first therapeutic class 


\begin{tabular}{|c|c|c|}
\hline Exclusion criteria $^{\mathrm{a}}$ & $\mathbf{n}$ & $\%$ \\
\hline Aged $\leq 18$ years & 23,171 & 1.5 \\
\hline Not eligible for medical and pharmacy services during 6-month pre-index period through 2-year post-index period & 994,027 & 62.2 \\
\hline Receipt of antidiabetic medication during pre-index period & 348,166 & 21.8 \\
\hline No evidence of receipt of antidiabetic medication during post-index period & 828,337 & 51.8 \\
\hline Incomplete ICD-9-CM codes without evidence of NIAD medication & 16,912 & 1.1 \\
\hline Diagnosis of type I diabetes mellitus anytime during the study period & 191,110 & 12.0 \\
\hline Diagnosis of gestational diabetes anytime during the study period & 11,753 & 0.7 \\
\hline Diagnosis of pregnancy anytime during the study period & 22,008 & 1.4 \\
\hline Target sample & 110,358 & 6.9 \\
\hline \multicolumn{3}{|l|}{ Treatment patterns } \\
\hline Noninsulin users & 103,138 & 93.5 \\
\hline Insulin-only users & 1,712 & 1.6 \\
\hline Early insulin users & 1,186 & 1.1 \\
\hline Same-day insulin users & 1,049 & 1.0 \\
\hline Short-term insulin users & 1,242 & 1.1 \\
\hline Intermittent insulin users & 296 & 0.3 \\
\hline Long-term insulin users & 1,735 & 1.6 \\
\hline
\end{tabular}

switch/add-on and end of the 24-month period, was used to calculate outcomes (Figure 1). The study cohorts were then matched in a ratio of 1:3 (insulin-use:NIAD-only). Matching was done using the technique of nearest available match on peri-period T2DM-specific costs $( \pm \$ 100)$, time to treatment switch/add-on ( \pm 1 day), presence of T2DM-specific hospital/ER visit during the peri-period (exact), and estimated propensity score (caliper width of \pm 0.001 unit). The greedy nearest-neighbor matching technique was employed to form pairs of insulin-use:NIAD-only cohorts matched on the logit of the propensity score. The propensity score for a patient was defined as the probability of being in the insulin-use cohort conditional on the following covariates measured during the pre-index period: patient age; gender; geographic region (categorized as Northeast, Midwest, South, and West); number of prescriptions; number of unique prescription classes; number of unique diagnoses; presence of 10 comorbidities (which included depression, anxiety, hypertension, obesity, cardiovascular disease [CVD], cerebrovascular disease, neuropathy, nephropathy, retinopathy, and peripheral vascular disease); and Charlson Comorbidity Index (CCI) score. ${ }^{18-20}$

The primary outcomes of interest were T2DM-specific resource utilization and costs, computed on a per-month basis, and hypoglycemia rates, which were captured during the outcomes assessment period. T2DM-specific resource utilization was identified by counting unique dates of service for T2DM (selected if diagnosis of T2DM was coded in the first or second position of the medical claim). Resource utilization was classified according to the place of service and included the proportion of patients with hospitalizations, ER visits, physician office visits, or other visits. T2DM-specific cost components included pharmacy and medical costs. Pharmacy costs included allowed amounts for all antidiabetic prescription claims and claims for insulin supplies, such as needles, strips, and lancets. Medical costs included allowed amounts for all T2DM-specific resource utilization. Similarly, all-cause medical and pharmacy costs were also calculated by summing allowed amounts for all medical and pharmacy claims, respectively. All costs were adjusted to 2010 U.S. dollars using the medical component of the Consumer Price Index. Hypoglycemia rates were captured by identifying medical claims with an ICD-9-CM primary or secondary diagnosis code for hypoglycemia based on an algorithm proposed in a previous study. ${ }^{21}$ The hypoglycemia incidence rate, captured during the outcomes assessment period, was calculated as the total number of hypoglycemic events divided by the total number of person-years (PYs) of follow-up and expressed as events per 100 PYs.

\section{Statistical Analysis}

Before matching, baseline characteristics were compared using t-tests for continuous variables and chi-square tests for categorical variables. After matching, baseline characteristics were compared using paired t-tests for continuous variables, and categorical variables were compared using McNemar's test. The 
TABLE 3 Baseline Description of Study Sample After Matching

\begin{tabular}{|c|c|c|c|c|c|c|}
\hline Characteristics & \multicolumn{2}{|c|}{$\begin{array}{c}\text { Insulin Use } \\
(\mathbf{n}=350)\end{array}$} & \multicolumn{2}{|c|}{$\begin{array}{l}\text { NIAD-Only }{ }^{\mathrm{a}} \\
(\mathrm{n}=1,050)\end{array}$} & $P$ Value $^{\mathrm{b}}$ & $\begin{array}{l}\text { Standardized } \\
\text { Difference }^{c}\end{array}$ \\
\hline \multicolumn{7}{|l|}{ Demographic characteristics } \\
\hline Age, years, mean (SD) & 56.33 & (12.8) & 55.66 & $(11.6)$ & 0.1986 & 5.5 \\
\hline Women, n (\%) & 133 & $(38.0)$ & 449 & $(42.8)$ & 0.0316 & 9.7 \\
\hline \multicolumn{7}{|l|}{ Geographic region, $\mathbf{n}(\%)$} \\
\hline East & 67 & $(19.1)$ & 188 & $(17.9)$ & \multirow{4}{*}{0.1464} & 3.2 \\
\hline Midwest & 169 & $(48.3)$ & 484 & $(46.1)$ & & 4.4 \\
\hline South & 63 & (18.0) & 208 & (19.8) & & 4.6 \\
\hline West & 51 & $(14.6)$ & 170 & $(16.2)$ & & 4.5 \\
\hline \multicolumn{7}{|l|}{ Index year (2003-2008), n (\%) } \\
\hline 2003 & 26 & $(7.4)$ & 96 & $(9.1)$ & \multirow{6}{*}{0.1785} & 6.2 \\
\hline 2004 & 59 & $(16.9)$ & 166 & (15.8) & & 2.8 \\
\hline 2005 & 64 & (18.3) & 187 & $(17.8)$ & & 1.2 \\
\hline 2006 & 74 & $(21.1)$ & 187 & $(17.8)$ & & 8.4 \\
\hline 2007 & 104 & $(29.7)$ & 344 & $(32.8)$ & & 6.6 \\
\hline 2008 & 23 & $(6.6)$ & 70 & $(6.7)$ & & 0.4 \\
\hline \multicolumn{7}{|l|}{ Comorbidity in pre-index period } \\
\hline Number of unique Rx classes, mean (SD) & 2.97 & $(4.3)$ & 2.88 & (3.8) & 0.6093 & 2.2 \\
\hline Number of Rx, mean (SD) & 3.13 & $(4.6)$ & 3.04 & $(4.1)$ & 0.6537 & 1.9 \\
\hline Number of unique Dx, mean (SD) & 4.87 & $(5.9)$ & 4.64 & (5.3) & 0.3586 & 3.9 \\
\hline CCI, mean (SD) & 0.30 & $(0.7)$ & 0.23 & $(0.7)$ & 0.0216 & 10.1 \\
\hline \multicolumn{7}{|l|}{ Other complications, $\mathbf{n}(\%)$} \\
\hline Depression & 12 & $(3.4)$ & 48 & $(4.6)$ & 0.2242 & 5.8 \\
\hline Anxiety & 7 & $(2.0)$ & 21 & $(2.0)$ & 1.0000 & 0.0 \\
\hline Hypertension & 84 & $(24.0)$ & 252 & $(24.0)$ & 1.0000 & 0.0 \\
\hline Obesity & 10 & $(2.9)$ & 35 & (3.3) & 0.6147 & 2.7 \\
\hline Cardiovascular disease & 39 & $(11.1)$ & 76 & $(7.2)$ & 0.0030 & 13.5 \\
\hline Nephropathy & 2 & $(0.6)$ & 5 & $(0.5)$ & 1.0000 & 1.3 \\
\hline Time to first treatment (in days), mean (SD) & 146 & $(175.0)$ & 153 & $(195.0)$ & 0.2755 & 3.6 \\
\hline Time to treatment switch/add on (in days), mean (SD) & 115 & $(145.0)$ & 115 & $(144.0)$ & 0.0600 & 0.0 \\
\hline Peri-period T2DM-specific costs, mean (SD), \$ & 152 & $(245.0)$ & 150 & $(243.0)$ & 0.2040 & 0.5 \\
\hline T2DM-specific hospital/ER visits in peri-period, $n$ (\%) & 0 & $(0.0)$ & 0 & $(0.0)$ & - & - \\
\hline
\end{tabular}

T2DM-specific hospital/ER visits in peri-period, $\mathbf{n}(\%)$

Note: Bold $P$ values indicate significance $(P<0.05)$

aPatients receiving 2 or more classes of NIADs.

${ }^{b} \mathrm{McNemar}$ 's test for dichotomous variables and paired t-test for continuous variables.

'Standardized difference $=100 \times(x 1-x 2) / \sqrt{ }\left\{\left(s 1^{2}+s 2^{2}\right) / 2\right\}$, where $x 1=$ mean of group $1, x 2=$ mean of group 2 , s1 $=$ standard deviation of group 1 , and $s 2=$ standard deviation of group 2.

$C C I=$ Charlson Comorbidity Index; Dx=diagnoses; ER=emergency room; NIAD=noninsulin antidiabetic; $R x=$ prescription; SD=standard deviation; T2DM = type 2 diabetes mellitus.

success of the matching process was also evaluated by assessing standardized differences in the covariates between the 2 cohorts. The standardized difference is the absolute difference in the means of a covariate across the groups divided by an estimate of the pooled standard deviation (SD) of the covariate. It is expressed as a percentage of pooled SDs. A standardized difference of $<10 \%$ was considered an acceptable level of difference. ${ }^{22}$ Differences in resource utilization and costs between the cohorts of interest were evaluated using multivariable logistic and gamma regressions, respectively. Only variables that showed significant between-group differences after the matching procedure were included in the multivariate models.
Negative binomial regression models were used to investigate differences in hypoglycemia rates between the cohorts, and the corresponding incident rate ratios (IRRs) and 95\% confidence intervals (CIs) were reported. All statistical analyses were conducted using SAS version 9.2 (SAS Institute, Cary, NC), with an a priori significance level of $\alpha=0.05$.

\section{Results}

\section{Demographics and Treatment Patterns}

A total of 110,358 patients (7\%) of the initially identified target population met all study criteria (Table 2). Most patients were noninsulin users (94\%; $n=103,138)$; approximately $2 \%$ of 


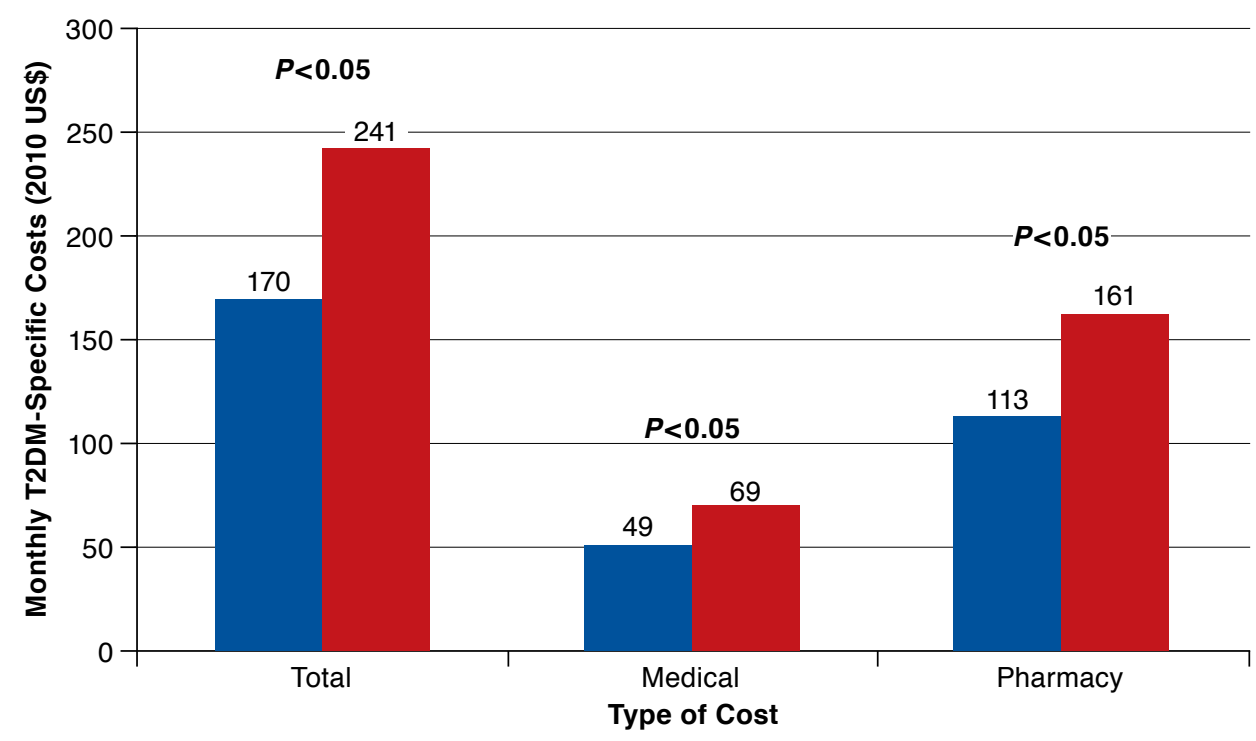

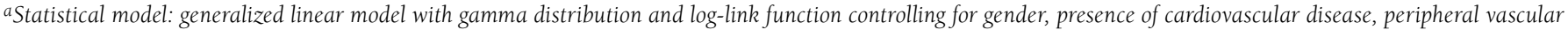
disease, and Charlson Comorbidity Index scores.

NIAD = noninsulin antidiabetic; T2DM= type 2 diabetes mellitus.

patients received only insulin; and another approximately 2\% were long-term insulin users ( $\mathrm{n}=1,735)$. Among the noninsulin users, the most commonly used NIAD class was biguanides (44\%), followed by sulfonylureas (9\%). In patients classified as long-term insulin users, the most common treatment regimen was initiation with biguanides and then switching to/adding on insulin (16.6\%).

To achieve the study objectives, patients on NIAD therapy who switched to/added a second NIAD therapy $(n=35,833)$ were selected from the noninsulin therapy users $(n=103,138)$, and patients on NIAD therapy who switched to/added on insulin $(n=758)$ were selected from the long-term insulin users $(n=1,735)$. This group constituted the final population. Accordingly, a total of 35,833 patients were categorized into the NIAD-only cohort, and 758 were categorized into the insulinuse cohort. Between the 2 cohorts of interest (insulin-use cohort and NIAD-only cohort), pre-match demographics were similar with respect to age and proportion of women; however, they were dissimilarly distributed with respect to geographic region, index year of T2DM diagnosis, $\mathrm{CCI}$ score, and diseaseseverity proxy measures (see Appendix A, available in online article). On average, both cohorts initiated treatment with NIAD within 4 months of index diagnosis; however, patients in the insulin-use cohort switched/added on therapy sooner than patients in the NIAD-only cohort (146 days vs. 167 days, respectively). After matching patients from the 2 study cohorts using propensity scores, 1,400 patients remained. Based on the 1:3 matching ratio, the insulin-use cohort included 350 patients, and the NIAD-only cohort included 1,050 patients. Table 3 describes the demographic and clinical characteristics of the study cohorts. The cohorts had similar demographic and clinical characteristics with SD values of $<10 \%$ for all baseline characteristics, except CCI scores and the proportion of patients with CVD (SD: CCI = 10.1, CVD = 13.5).

After matching, the adjusted T2DM-specific costs per month incurred during the outcomes assessment period were compared (Figure 2). The insulin-use cohort had significantly higher total costs (medical+pharmacy) of $\$ 241$ per month compared with those of the NIAD-only cohort of $\$ 170$ per month $(P=0.0003)$. The mean monthly T2DM-specific medical costs for the insulin-use cohort were $\$ 69$ (Figure 2). The higher medical costs were driven mainly by higher monthly physician visits ( $91 \%$ vs. $86 \%, P=0.0311$; Table 4 ). The mean monthly T2DM-specific pharmacy costs were also higher for the insulin-use cohort compared with the NIAD-only cohort (\$161/month vs. \$113/month). Similar trends were observed for all-cause costs (see Appendix B, available in online article). Furthermore, among matched patients in the insulin-use cohort, the rate of hypoglycemic events was 10.2 per 100 PYs versus 2.9 per 100 PYs in patients belonging to the NIAD-only cohort $(\mathrm{IRR}=3.85,95 \% \mathrm{CI}=0.03-0.31)$. 


\section{TABLE 4 T2DM-Specific Resource Utilization}

\begin{tabular}{|c|c|c|c|c|c|}
\hline $\begin{array}{l}\text { Type of Resource } \\
\text { Utilization, n (\%) }\end{array}$ & \multicolumn{2}{|c|}{$\begin{array}{l}\text { NIAD-Only }{ }^{\mathrm{a}} \\
(\mathrm{n}=1,050)\end{array}$} & \multicolumn{2}{|c|}{$\begin{array}{c}\text { Insulin Use } \\
(\mathbf{n}=350)\end{array}$} & $\begin{array}{c}P \\
\text { Valueb }^{b}\end{array}$ \\
\hline Inpatient hospital visits & 3 & $(0.3)$ & 2 & $(0.6)$ & 0.9183 \\
\hline ER visits & 47 & $(4.5)$ & 25 & (7.1) & 0.0849 \\
\hline Physician visits & 907 & $(86.4)$ & 317 & (90.6) & 0.0311 \\
\hline Outpatient visits & 1 & $(47.7)$ & 175 & $(50.0)$ & 0.4986 \\
\hline Otherc & 165 & $(15.7)$ & 56 & $(16.0)$ & 0.6733 \\
\hline \multicolumn{6}{|c|}{$\begin{array}{l}\text { Note: Bold P values indicate significance }(P<0.05) \text {. } \\
\text { aPatients receiving } 2 \text { or more classes of NIADs. } \\
{ }^{b} \text { Logistic regression controlling for female gender, presence of cardiovascular } \\
\text { disease, peripheral vascular disease, and Charlson Comorbidity Index score. } \\
\text { 'Home health care, durable medical equipment, and other inpatient stays (skilled } \\
\text { nursing facility and rehabilitation stays). } \\
\text { ER=emergency room; NIAD = noninsulin antidiabetic; T2DM= type } 2 \text { diabetes } \\
\text { mellitus. }\end{array}$} \\
\hline
\end{tabular}

\section{Discussion}

This study assessed health care utilization and costs associated with the use of insulin therapy among newly diagnosed patients with T2DM in a managed care setting. When compared with patients who did not switch to/add on insulin (NIAD-only cohort), patients who switched to/added on insulin (insulin-use cohort) had a higher number of hypoglycemic events and were more likely to utilize health care resources due to T2DM and, therefore, had higher T2DM-specific cost outcomes. Specifically, the insulin-use cohort utilized a significantly higher number of health care resources (physician visits) compared with the NIAD-only cohort. Furthermore, the insulin-use cohort incurred approximately $\$ 70$ per month more in T2DM-specific costs compared with the NIAD-only cohort.

Previous studies have shown equivalence in clinical outcomes between patients using insulin versus NIAD therapy. ${ }^{5-7,23-25}$ Schwartz et al. (2003) found similar Alc and fasting plasma glucose levels in patients using triple oral antidiabetic therapy versus a combination of metformin and insulin (70/30) after 24 weeks of therapy. ${ }^{23}$ Another study found that triple oral antidiabetic therapy versus insulin plus metformin resulted in clinically equivalent outcomes and did not affect compliance, treatment satisfaction, or quality of life. ${ }^{5}$ Data from other studies also showed comparable results in terms of glycemic control when adding insulin versus another oral antidiabetic drug. ${ }^{6}$ Additionally, 2 studies showed that the mean effect of insulin on Alc does not seem to be greater than that of other medications, such as sulfonylureas, when combined with metformin. ${ }^{24,25}$

Real-world studies, on the other hand, showed divergent results, wherein use of insulin in T2DM was associated with poor clinical outcomes compared with use of NIAD therapy only. ${ }^{26,27}$ A study evaluating a cohort from the Veterans Health
Administration, Medicare, and National Death Index databases, retrospectively, found that patients with T2DM who added insulin to metformin had an increased risk of nonfatal cardiovascular outcomes and all-cause mortality compared with patients who added a sulfonylurea to metformin. ${ }^{26}$ Another retrospective cohort study conducted using the United Kingdom General Practice Research Database from 2000 to 2010 found that insulin use was associated with an increased risk of diabetes-related complications and all-cause mortality in patients with T2DM versus other regimens that did not contain insulin (e.g., metformin monotherapy, sulfonylurea monotherapy, or metformin + sulfonylurea combination therapy). ${ }^{27}$

Given the divergent results when comparing insulin regimens and oral antidiabetic therapy regimens in terms of clinical outcomes, it is prudent to evaluate costs and health care resource utilization in order to quantify the economic burden of these treatment alternatives in patients with T2DM. A study by Waugh et al. (2010) found that regimens containing insulin were more expensive in terms of direct costs than such thiazolidinediones as pioglitazone and rosiglitazone but had similar effects in controlling plasma glucose. ${ }^{17}$ This study similarly found significantly higher T2DM-specific costs for patients using insulin compared with oral antidiabetic drugs but was limited by the nature of the data source in evaluating glycemic control. However, this study was able to assess the impact on hypoglycemic events and found the use of oral antidiabetic medications to be associated with a lower risk of hypoglycemia compared with insulin use, similar to findings from another study. ${ }^{28}$ Curkendall et al. (2011) showed lower hypoglycemic event rates in patients receiving NIAD regimens (sulfonylurea: 4/100 PYs; nonsulfonylurea NIAD: 1.9/100 PYs) compared with patients receiving insulin plus NIAD regimens (insulin + sulfonylurea: 9.2/100 PYs; insulin + nonsulfonylurea NIAD: 6.5/100 PYs). ${ }^{28}$ Thus, this analysis demonstrates that using noninsulin antidiabetic medications result in lower costs and resource use compared with using insulin and avoids outcomes such as hypoglycemia that could substantially affect the health care system and patient quality of life.

\section{Limitations}

The following limitations must be considered when interpreting these results. Patients who had incomplete ICD-9-CM codes of 250, which makes it difficult to determine whether they have type 1 diabetes mellitus or T2DM, were not included in the study. In addition, there may be errors in the coding of claims or diagnoses. This may attenuate the generalizability of the study; however, results indicate that only approximately $1 \%$ of the patients had incomplete ICD-9-CM codes. Hence, it is unlikely that the effects of exclusion would be substantial. 
There are also inherent limitations to a database study that may lead to gaps in information gathered, limit evaluation to claims within the study period, and do not allow evaluation of patient compliance with medications. Although this study evaluated patients based on a date of first diagnosis and subsequent receipt of NIAD therapy, it is possible that patients enrolled in the study may have been treated with other/additional NIADs or insulin therapy before the start of the study period. Limitations in the ability to accurately assess patient compliance may result in attributing certain health care utilization or medical costs to therapy.

From a data capability perspective, this dataset represents predominantly a managed care population that may be different from other populations, and because of the time lag associated with this dataset, the study period may not reflect current practices. The analysis controlled for the majority of differences in baseline characteristics using propensity score matching, with the exception of baseline CCI scores and CVD. In addition, residual confounding may also exist due to lack of information on clinical measures such as Alc and body mass index, which may differ between cohorts. Alc, in particular, is an important factor, as it characterizes disease severity, which, in turn, may have driven a particular treatment regimen or may have accounted for the differences in hypoglycemic events. Therefore, differences among cohorts in baseline CCI scores, CVD, and residual confounders may have affected health care resource utilization and/or costs. To account for some of the residual confounding due to differences in disease severities between the study cohorts, the analysis controlled for disease-severity proxy measures such as peri-period T2DMspecific costs and rates of hospital/ER visits. As a result of this approach, only patients without T2DM-specific hospital/ER visits in the peri-period were included in the analysis after the match-this aspect may affect the generalizability of the findings. Furthermore, these data also pose a potential limitation of misclassification of patient types, particularly the insulin-use cohort, based on variables such as days' supply. This limitation was addressed by testing the cohort definition using other available data elements, such as number of prescriptions filled.

\section{Conclusions}

Use of insulin therapy within a population newly diagnosed with T2DM was associated with increased hypoglycemic events, T2DM-specific pharmacy and medical costs, and utilization of health care services. Future research should focus on the impact of clinical parameters (e.g., Alc) on outcomes associated with patients on NIAD therapies with or without the addition of insulin. Additionally, further investigation on how hypoglycemic events (particularly severe events) affect glucose control and patient compliance is warranted.

\section{Authors}

KELLY BELL, PharmD, MSPhr, is HEOR Director, U.S. Medical Affairs, AstraZeneca, Wilmington, Delaware, and SHREEKANT PARASURAMAN, PhD, is Senior Director, Incyte Corporation, Wilmington, Delaware. ADITYA RAJU, BPharm, MS, is Manager, Applied Data Analytics, and MELISSA DENNO, PharmD, MS, is Assistant Director, Global Health Economics \& Outcomes Research, Xcenda, Palm Harbor, Florida. MANAN SHAH, PharmD, PhD, is Director, Health Services \& Outcomes Research, Bristol-Myers Squibb, Plainsboro, New Jersey, and JOHN GRAHAM, PharmD, is Vice President, Global VEO, GlaxoSmithKline Pharmaceuticals, Collegeville, Pennsylvania.

AUTHOR CORRESPONDENCE: Melissa Denno, PharmD, MS, Xcenda, LLC, 4114 Woodlands Pkwy., Ste. 500, Palm Harbor, FL 34685. Tel.: 727.771.4167; E-mail: melissa.denno@xcenda.com.

\section{DISCLOSURES}

This work was supported by Bristol-Myers Squibb and AstraZeneca.

Denno and Raju are employees of Xcenda, which has received research funding from Bristol-Myers Squibb. At the time of this research, Bell and Graham were employees of Bristol-Myers Squibb; Bell has stock in BristolMyers Squibb. Parasuraman was an employee of AstraZeneca, and Shah was an employee of Xcenda.

Study concept and design were contributed by Bell, Shah, Raju, Graham, and Parasuraman. Data collection was performed by Raju, Shah, Parasuraman, and Bell, and analysis was carried out by Parasuraman, Graham, Shah, Raju and Bell. The manuscript was written by Denno, Bell, Parasuraman, Raju, and Shah, assisted by Graham, and revised by Denno, Raju, Bell, Parasuraman, and Shah.

\section{REFERENCES}

1. American Diabetes Association. Economic costs of diabetes in the U.S. in 2012. Diabetes Care. 2013;36(4):1033-46.

2. Asche C, LaFleur J, Conner C. A review of diabetes treatment adherence and the association with clinical and economic outcomes. Clin Ther. 2011;33(1):74-109.

3. American Diabetes Association. Standards of medical care in diabetes-2012. Diabetes Care. 2012;35(Suppl 1):S11-S63.

4. Handelsman Y, Mechanick J, Blonde L, et al. American Association of Clinical Endocrinologists medical guidelines for clinical practice for developing a diabetes mellitus comprehensive care plan. Endocr Pract. 2011;17(Suppl 2):1-53.

5. Lingvay I, Legendre JL, Kaloyanova PF, et al. Insulin-based versus triple oral therapy for newly diagnosed type 2 diabetes. Diabetes Care. 2009;32(10):1789-95

6. Gamble JM, Simpson SH, Brown LC, et al. Insulin versus an oral antidiabetic agent as add-on therapy in type 2 diabetes after failure of an oral antidiabetic regimen: a meta-analysis. Open Med. 2008;2(2):e26-e38.

7. McIntosh B, Cameron C, Singh SR, et al. Second-line therapy in patients with type 2 diabetes inadequately controlled with metformin monotherapy: a systematic review and mixed-treatment comparison meta-analysis. Open Med. 2011;5(1):e35-e48.

8. Henderson JN, Allen KV, Deary IJ, et al. Hypoglycaemia in insulin-treated type 2 diabetes: frequency, symptoms and impaired awareness. Diabet Med. 2003;20(12):1016-21. 


\section{Resource Utilization and Costs Associated with Using Insulin Therapy Within a Newly Diagnosed Type 2 Diabetes Mellitus Population}

9. Sarkar U, Karter AJ, Liu JY, et al. Hypoglycemia is more common among type 2 diabetes patients with limited health literacy: the Diabetes Study of Northern California (DISTANCE). J Gen Intern Med. 2010;25(9):962-68.

10. Bennett WL, Wilson LM, Bolen S, et al. Oral diabetes medications for adults with type 2 diabetes: an update. Comparative Effectiveness Review No. 27. (Prepared by Johns Hopkins University Evidence-based Practice Center under Contract No. 290-02-0018.) AHRQ Publication No. 11-EHC038-EF. Rockville, MD: Agency for Healthcare Research and Quality. March 2011. Available at: http://effectivehealthcare.ahrq.gov/ehc/ products/155/644/type-2-diabetes-medications-report-130911.pdf. Accessed February 1, 2015.

11. Budnitz DS, Lovegrove MC, Shehab N, et al. Emergency hospitalizations for adverse drug events in older Americans. N Engl J Med. 2011;365(21):2002-12.

12. Moghissi ES, Korytkowski MT, DiNardo M, et al. American Association of Clinical Endocrinologists and American Diabetes Association consensus statement on inpatient glycemic control. Diabetes Care. 2009;32(6):1119-31.

13. Turchin A, Matheny ME, Shubina M, Scanlon JV, Greenwood B, Pendergrass ML. Hypoglycemia and clinical outcomes in patients with diabetes hospitalized in the general ward. Diabetes Care. 2009;32(7):1153-57.

14. Krinsley JS, Grover A. Severe hypoglycemia in critically ill patients: risk factors and outcomes. Crit Care Med. 2007;35(10):2262-67.

15. Cook CB, Jameson KA, Hartsell ZC, et al. Beliefs about hospital diabetes and perceived barriers to glucose management among inpatient midlevel practitioners. Diabetes Educ. 2008;34(1):75-83.

16. American Diabetes Association. Standards of medical care in diabetes2011. Diabetes Care. 2011;34(Suppl 1):S11-S61.

17. Waugh N, Cummins E, Royle P, et al. Newer agents for blood glucose control in type 2 diabetes: systematic review and economic evaluation. Health Technol Assess. 2010;14(36):1-248.

18. Deyo RA, Cherkin DC, Ciol MA. Adapting a clinical comorbidity index for use with ICD-9-CM administrative databases. J Clin Epidemiol. 1992;45(6):613-19.
19. D'Hoore W, Bouckaert A, Tilquin C. Practical considerations on the use of the Charlson comorbidity index with administrative databases. J Clin Epidemiol. 1996;49(12):1429-33.

20. Romano PS, Roos LL, Jollis JG. Adapting a clinical comorbidity index for use with ICD-9-CM administrative data: differing perspectives. J Clin Epidemiol. 1993;46(10):1075-79.

21. Ginde AA, Blanc PG, Lieberman RM, et al. Validation of ICD-9-CM coding algorithm for improved identification of hypoglycemia visits. BMC Endocr Disord. 2008;8:4.

22. Austin PC. A critical appraisal of propensity-score matching in the medical literature between 1996 and 2003. Statist Med. 2008;27(12):2037-49.

23. Schwartz S, Sievers R, Strange P, Lyness WH, Hollander P; INS-2061 Study Team. Insulin 70/30 mix plus metformin versus triple oral therapy in the treatment of type 2 diabetes after failure of two oral drugs. Diabetes Care. 2003;26(8):2238-43.

24. Monami M, Lamanna C, Marchionni N, Mannucci E. Comparison of different drugs as add-on treatments to metformin in type 2 diabetes: a metaanalysis. Diabetes Res Clin Pract. 2008;79(2):196-203.

25. Malone JK, Beattie SD, Campaigne BN, Johnson PA, Howard AS, Milicevic Z. Therapy after single oral agent failure: adding a second oral agent or an insulin mixture? Diabetes Res Clin Pract. 2003;62(3):187-95.

26. Roumie CL, Greevy RA, Grijalva CG, et al. Association between intensification of metformin treatment with insulin vs sulfonylureas and cardiovascular events and all-cause mortality among patients with diabetes. JAMA. 2014;311(22):2288-96.

27. Currie CJ, Poole CD, Evans M, Peters JR, Morgan CL. Mortality and other important diabetes-related outcomes with insulin vs other antihyperglycemic therapies in type 2 diabetes. J Clin Endocrinol Metab. 2013;98(2):668-77.

28. Curkendall SM, Zhang B, Oh KS, Williams SA, Pollack MF, Graham J. Incidence and cost of hypoglycemia among patients with type 2 diabetes in the United States: analysis of a health insurance database. J Clin Outcomes Manage. 2011;18(10):455-62. 


\section{Resource Utilization and Costs Associated with Using Insulin Therapy Within a Newly Diagnosed Type 2 Diabetes Mellitus Population}

APPENDIX A Baseline Description of Study Sample Before Match

\begin{tabular}{|c|c|c|c|c|c|c|}
\hline Characteristics & \multicolumn{2}{|c|}{$\begin{array}{c}\text { Insulin Use } \\
(\mathrm{N}=758)\end{array}$} & \multicolumn{2}{|c|}{$\begin{array}{l}\text { NIAD-Onlya } \\
(\mathrm{N}=35,833)\end{array}$} & $P$ Value ${ }^{b}$ & $\begin{array}{l}\text { Standardized } \\
\text { Difference }^{c}\end{array}$ \\
\hline \multicolumn{7}{|l|}{ Demographic characteristics } \\
\hline Age, years, mean (SD) & 55.14 & $(12.4)$ & 54.54 & $(11.3)$ & 0.1836 & 5.1 \\
\hline Female, n (\%) & 332 & $(43.8)$ & 15,918 & $(44.4)$ & 0.7325 & 1.3 \\
\hline \multicolumn{7}{|l|}{ Geographic region, $\mathbf{n}(\%)$} \\
\hline East & 110 & $(14.5)$ & 9,826 & $(27.4)$ & \multirow{4}{*}{$<0.0001$} & 32.1 \\
\hline Midwest & 371 & $(48.9)$ & 14,613 & $(40.8)$ & & 16.5 \\
\hline South & 146 & (19.3) & 6,261 & $(17.5)$ & & 4.6 \\
\hline West & 131 & $(17.3)$ & 5,133 & $(14.3)$ & & 8.1 \\
\hline \multicolumn{7}{|l|}{ Index year (2003-2008), n (\%) } \\
\hline 2003 & 48 & $(6.3)$ & 2,990 & $(8.3)$ & \multirow{6}{*}{$<0.0001$} & 7.7 \\
\hline 2004 & 102 & $(13.5)$ & 6,076 & $(17.0)$ & & 9.8 \\
\hline 2005 & 127 & (16.8) & 6,582 & (18.4) & & 4.2 \\
\hline 2006 & 134 & $(17.7)$ & 7,698 & $(21.5)$ & & 9.6 \\
\hline 2007 & 286 & $(37.7)$ & 10,335 & $(28.8)$ & & 18.9 \\
\hline 2008 & 61 & $(8.1)$ & 2,152 & $(6.0)$ & & 8.0 \\
\hline \multicolumn{7}{|l|}{ Comorbidity in pre-index period, mean (SD) } \\
\hline Number of unique Rx classes & 2.96 & $(4.2)$ & 3.13 & $(3.7)$ & 0.2777 & 4.2 \\
\hline Number of Rxs & 3.15 & $(4.6)$ & 3.29 & $(4.0)$ & 0.4062 & 3.3 \\
\hline Number of unique diagnoses & 4.87 & $(5.8)$ & 4.96 & $(5.3)$ & 0.6632 & 1.7 \\
\hline CCI & 0.41 & $(1.0)$ & 0.28 & $(0.8)$ & 0.0003 & 14.8 \\
\hline Time to first treatment (in days), mean (SD) & 108 & (154) & 110 & $(170)$ & 0.6524 & 1.6 \\
\hline Time to treatment switch/add on (in days), mean (SD) & 146 & $(172)$ & 167 & $(195)$ & 0.0010 & 11.3 \\
\hline Peri-period T2DM-specific costs, mean (SD), \$ & 1,223 & $(5,432)$ & 628 & $(5,036)$ & 0.0029 & 11.4 \\
\hline Disease-specific hospital/ER visits in peri-period, $\mathbf{n}(\%)$ & 120 & $(15.8)$ & 2,410 & $(6.7)$ & $<0.0001$ & 29.1 \\
\hline \multicolumn{7}{|c|}{$\begin{array}{l}\text { Note: Bold P values indicate significance }(P<0.05) \text {. } \\
\text { aPatients receiving } 2 \text { or more classes of NIADs. } \\
\text { bChi-square test for dichotomous variables and } t \text {-test for continuous variables. } \\
\text { 'Standardized difference }=100 \times(x 1-x 2) / \sqrt{ }\left\{\left(s 1^{2}+s 2^{2}\right) / 2\right\} \text {, where } x 1=\text { mean of group } 1, x 2=\text { mean of group } 2, s 1=s t a n d a r d \text { deviation of group } 1 \text {, and } s 2=\text { standard deviation } \\
\text { of group } 2 \text {. } \\
\text { CCI=Charlson Comorbidity Index; ER=emergency room; NIAD=noninsulin antidiabetic; Rx=prescription; } S D=\text { standard deviation; T2DM=type } 2 \text { diabetes mellitus. }\end{array}$} \\
\hline
\end{tabular}

\begin{tabular}{|c|c|c|c|c|c|}
\hline APPEN & K & $\begin{array}{l}\text { Monthly } \\
\text { Costs by }\end{array}$ & $\begin{array}{l}\text { juste } \\
\text { udy }\end{array}$ & $\begin{array}{l}\text { All-Cause } \\
\text { hort }\end{array}$ & \\
\hline $\begin{array}{l}\text { All-Cause } \\
\text { Costs Mean } \\
(95 \% \text { CI })\end{array}$ & & $\begin{array}{l}\text { D-Onlya } \\
=1,050)\end{array}$ & & $\begin{array}{l}\text { alin Use } \\
=350)\end{array}$ & $P$ Value $^{b}$ \\
\hline Total (\$) & 887 & $(640-1,353)$ & 1,283 & $(916-2,003)$ & $<0.0001$ \\
\hline Medical (\$) & 548 & $(355-1,029)$ & 795 & $(508-1,555)$ & 0.0002 \\
\hline Pharmacy (\$) & 338 & $(253-483)$ & 486 & $(362-705)$ & 0.0005 \\
\hline
\end{tabular}

Note: Bold $P$ values indicate significance $(P<0.05)$.

a Patients receiving 2 or more classes of NIADs.

${ }^{b}$ Generalized linear model with gamma distribution and log-link function control-

ling for gender, presence of cardiovascular disease, peripheral vascular disease, and

Charlson Comorbidity Index scores.

$C I=$ confidence interval; NIAD = noninsulin antidiabetic. 